\title{
Distribución de Taxus globosa (Taxaceae) en México: Modelos ecológicos de nicho, efectos del cambio del uso de suelo y conservación
}

\author{
Distribution of Taxus globosa (Taxaceae) in Mexico: Ecological niche modeling, \\ effects of land use change and conservation
}

\author{
RAÚL CONTRERAS-MEDINA ${ }^{1,2}$, ISOLDA LUNA-VEGA ${ }^{1, ~}{ }^{*}$ \& CÉSAR A. RÍOS-MUÑOZ1 \\ ${ }^{1}$ Departamento de Biología Evolutiva, Facultad de Ciencias, Universidad Nacional Autónoma de México, Apartado Postal \\ 70-399, México, DF, C. P. 04510, México \\ ${ }^{2}$ Escuela de Biología, Facultad de Ciencia y Tecnología, Universidad Simón Bolívar, Av. Río Mixcoac 48, col. Insurgentes \\ Mixcoac, México, DF, C. P. 03920, México \\ *Autor correspondiente: ilv@hp.fciencias.unam.mx
}

\begin{abstract}
RESUMEN
Taxus globosa es una gimnosperma que habita principalmente en los bosques templados de México y el norte de América Central, y que está considerada como amenazada por las agencias de conservación tanto mexicanas como internacionales. En México, una de las mayores amenazas para la mayoría de las especies de los bosques nublados es la pérdida de hábitat, la cual se propicia por la modificación de la cobertura vegetal por factores antropogénicos. Con base en 73 registros de herbario se obtuvo la distribución potencial de la especie mediante el algoritmo Maxent. Asimismo, a partir del modelo obtenido se analizó la modificación de la cobertura vegetal durante tres períodos (1976, 1996 y 2000) y su influencia en la distribución potencial de esta especie. Su distribución potencial fue evaluada en relación con el actual Sistema Nacional de Áreas Naturales Protegidas a nivel federal (ANPs) de México y las Regiones Terrestres Prioritarias para la Conservación (RTPs), con el fin de determinar el papel de estas áreas en su conservación y manejo. Los modelos de distribución potencial muestran que existe una reducción importante del área de distribución (hasta el $84 \%$ ) al existir un cambio de uso de suelo. La especie está mal representada en las ANPs, pues menos del $10 \%$ de su área potencial se encuentra dentro de estas áreas de protección federal. Este tipo de análisis representa una herramienta importante en biogeografía y conservación, así como en otras áreas del quehacer biológico, debido a su habilidad predictiva. Nuestros resultados muestran la urgencia que existe de conservar algunos parches de bosques templados mexicanos (principalmente los bosques nublados) para garantizar la existencia de esta especie.
\end{abstract}

Palabras clave: áreas naturales protegidas, distribución potencial, Maxent, romerillo.

\begin{abstract}
Taxus globosa is a gymnosperm inhabiting Mexican and northern Central American temperate forests, and it has been considered as threatened by Mexican and international conservancy agencies. In Mexico, habitat loss is one of the main threats for most of the cloud forest species including this taxon, being human-induced land use change a key issue. We obtained the potential distribution of this species through Maxent algorithm based on 73 herbarium records. Also the modification of vegetation cover for three time periods (1976, 1996 y 2000$)$ was analyzed upon the potential distribution of this species. The known and potential geographical distribution was evaluated in relation to the current Mexican National System of Natural Protected Areas at federal level (ANPs) and Mexican Priority Regions for Conservation (RTPs), in order to determine the role of these areas in its conservation and management. Potential distribution models show an important reduction (until $84 \%$ ) of the distribution area related with land use change. This species is scarcely represented in the ANPs, because less than $10 \%$ of its potential distribution occurs within these areas of federal protection. This approach represents an important tool in biogeography and conservation, as well as in other areas of biology, due to its predictive ability. Our results highlight the urgency to preserve the remaining Mexican temperate forest patches, mainly cloud forest ones, to guarantee the existence of this species.
\end{abstract}

Key words: Maxent, Mexican yew, natural protected areas, predicted distribution.

\section{INTRODUCCIÓN}

México ha sido considerado como uno de los países megadiversos a nivel mundial (Mittermeier \& Mittermeier 1992); la parte sur de México junto con el norte de América Central ha sido incluida por varios autores dentro de uno de los grandes "hotspots" de biodiversidad (Groombridge 1992, Heywood \& Davis 1997, Myers et al. 2000, Brooks et al. 
2001, Luna \& Contreras-Medina en prensa). No obstante lo anterior, solo existen algunos trabajos en los que se han delineado mapas de distribución, obtenidos a partir de un conjunto de puntos que representan las localidades donde los especímenes de una especie en particular ha sido colectada o registrada (Rapoport 1975, Skov 2000). Otros procedimientos utilizados para delinear la distribución geográfica de taxa mexicanos y que se han utilizados para diferentes estudios biogeográficos han sido los límites políticos (estados o municipios) (e.g., Villarreal et al. 1996, Dávila-Aranda et al. 2004) y celdas o cuadrículas de tamaño definido (grados o minutos de latitud y longitud, o hectáreas o kilómetros) (e.g., García-Mendoza 1995, Contreras-Medina \& Luna 2007). Sin embargo, aún es necesario obtener información sobre la distribución de ciertas especies consideradas como raras o amenazadas a partir de métodos formales y rigurosos (Engler et al. 2004). Collar (1996) sugirió que el solo hecho de que un taxón esté incluido bajo alguna categoría de riesgo debería llevarnos a su conservación, para garantizar su sobrevivencia y evitar su extinción.

En los últimos años se han desarrollado varios programas para realizar modelos ecológicos de nicho, como por ejemplo Bioclim (Nix 1986), GARP (Stockwell \& Peters 1999) y Maxent (Phillips et al. 2004), convirtiéndose en importantes herramientas en biología de la conservación y ecología del paisaje (Peterson \& Cohoon 1999). Estos modelos están basados en el concepto de nicho ecológico de Hutchinson (1957) relacionando la información biológica (puntual) con información ambiental (geográfica) y posteriormente identificando zonas donde no existan registros previos de la especie, corrigiendo la predicción y obteniendo de esta forma el área de distribución potencial de las especies (Peterson et al. 2002).

Estas aplicaciones han sido utilizadas recientemente para evaluar el impacto provocado por el cambio de uso de suelo (Sánchez-Cordero et al. 2005, Peterson et al. 2006, Ríos-Muñoz \& Navarro-Sigüenza 2009), que implica la transformación de la cobertura vegetal original por factores antropogénicos (Velázquez et al. 2002). El cambio de uso de suelo es una de las principales causas de la pérdida de biodiversidad, pues puede provocar la desaparición eventual de ciertas poblaciones dentro de las áreas de distribución de las especies (Peterson et al. 2006).

Actualmente son pocos los trabajos realizados bajo el enfoque del modelado de nicho ecológico con plantas mexicanas (Yberri 2009), entre los que se encuentran el de Téllez-Valdés et al. (2006) con Fagus grandifolia, Villaseñor \& Téllez-Valdés (2004) con el género Jefea y Golubov et al. (2005) con los géneros Agave y Opuntia, con la finalidad de delimitar el área de distribución geográfica; por otra parte se encuentran los de TéllezValdés \& Dávila-Aranda (2003) y GómezMendoza \& Arriaga (2007) con modelos de cambio climático basados en la distribución de cactáceas del Valle de Tehuacán-Cuicatlán y pinos y encinos de México, respectivamente, así como el de Solano \& Feria (2007) con el género Polianthes (Agavaceae) e Yberri (2009) con Nopalxochia phyllanthoides (Cactaceae), en los cuales se describen el estatus de riesgo y la distribución de ambos taxa. En todos los trabajos anteriores fueron utilizados los algoritmos Bioclim y GARP para generar las hipótesis de distribución potencial. Recientemente Maxent fue aplicado para evaluar la distribución de helechos y licofitas de la península de Yucatán (Ramírez-Barahona et al. 2009) y para llevar a cabo un estudio areográfico y de conservación del subgénero Echinoramaria (Gomphaceae) de México (González-Ávila et al. en prensa).

En el presente trabajo evaluamos la distribución de Taxus globosa Schltdl. (Taxaceae), conocida como "romerillo" o "granadillo" en México y como "Mexican yew” en América del Norte. Es un árbol bajo que crece en las laderas sombreadas como parte del estrato arbóreo bajo de algunos bosques templados, tales como los de abetos, pinos, encinos o bosques nublados, en un intervalo altitudinal que va de $\operatorname{los} 1000$ a los $2950 \mathrm{~m}$ sobre el nivel del mar. Esta planta ha sido registrada como poco común en muchas de las localidades donde ha sido recolectada (Contreras-Medina \& Luna 2001) y está incluida en la Norma Oficial Mexicana NOM059-ECOL-2001, que enlista las especies mexicanas bajo alguna categoría de riesgo (SEMARNAT 2002), bajo la categoría de sujeta a protección especial, la cual implica que se deben desarrollar programas de recuperación y conservación de las 
poblaciones naturales de la especie. La NOM059 (SEMARNAT 2002) es el documento oficial publicado por el gobierno mexicano para enlistar a las especies de seres vivos que habitan en su territorio y que se encuentran en alguna categoría de riesgo. Asimismo la especie está incluida en la categoría de bajo riesgo por la UICN (Luna et al. 2006).

Taxus globosa habita desde el noreste de México hasta el norte de América Central, en Guatemala, Honduras y El Salvador (Contreras-Medina \& Luna 2001). En México, la información detallada sobre su distribución geográfica y ecología es escasa y fragmentaria, a excepción de un estudio de una población del estado de San Luis Potosí (García \& Castillo 2000). Desafortunadamente, existe una creciente destrucción de los bosques donde habita la especie, mismos que han sido afectados severamente en los últimos años por las actividades humanas (Zamudio 1992).

En el presente estudio actualizamos el conocimiento que se tiene acerca de la distribución geográfica de Taxus globosa en México y con esta información generamos un modelo de distribución potencial en el país utilizando el algoritmo Maxent (Phillips et al. 2004). Asimismo, calculamos el impacto provocado por el cambio de uso de suelo en tres diferentes tiempos (1976, 1996 y 2000); por otra parte, contrastamos la distribución potencial de esta especie en relación con el sistema actual de Áreas Naturales Protegidas federales y Regiones Terrestres Prioritarias para la conservación en México, con la finalidad de verificar el papel de estas áreas en su conservación y manejo.

\section{MÉTODOS}

Los datos de distribución geográfica de Taxus globosa fueron obtenidos a partir de la consulta de ejemplares de los siguientes herbarios: Herbario Nacional del Instituto de Biología, UNAM (MEXU), Herbario de la Escuela Nacional de Ciencias Biológicas, IPN (ENCB), Herbario del Jardín Botánico de Missouri (MO), Herbarios del Instituto de Ecología A.C. en Xalapa (XAL) y en Pátzcuaro (IEB), Herbario de la Facultad de Ciencias, UNAM (FCME), Herbario de la Universidad de Guadalajara (IBUG), Herbario del Departamento de Bosques, Universidad Autónoma Chapingo (CHAP) y Herbario Nacional Forestal (INIF). Se obtuvieron un total de 73 registros (localidades únicas) que incluyen las coordenadas geográficas (latitud y longitud) y que fueron georreferenciadas a partir de mapas topográficos con escalas 1:50,000 y 1:250,000 producidos por el Instituto Nacional de Estadística, Geografía e Informática (INEGI).

Para obtener los modelos ecológicos de nicho se utilizó el algoritmo Maxent (Phillips et al. 2006), que ha sido evaluado y probado como uno de los mejores modeladores (Elith et al. 2006). El algoritmo se basa en datos de presencia conocida (puntos) y pseudoausencias (lugares en los que no existen datos puntuales). Está centrado en ajustar la distribución de la probabilidad de la ocurrencia de las especies al conjunto de píxeles a lo largo del área de estudio, basándose en la idea de que la mejor explicación a un fenómeno desconocido maximizará la entropía o la incertidumbre de la distribución de la probabilidad dependiendo de ciertas limitaciones, que en el caso de los modelos ecológicos de nicho, consisten en valores de aquellos píxeles en los cuales la especie ha sido detectada (Phillips et al. 2004, 2006). Las coberturas correspondientes a los parámetros ecológicos utilizados consistieron en 19 capas de información derivadas de temperatura y precipitación, provenientes de WorldClim 1.4 (Hijmans et al. 2005) y tres coberturas topográficas del proyecto Hydro1k (http:// eros.usgs.gov/\#/Find_Data/Products_and Data_Available/gtopo30/hydro), todas a una resolución espacial aproximada de $1 \mathrm{~km}^{2}$ (Tabla 1 ).

\section{TABLA 1}

\section{Variables geográficas utilizadas para obtener los modelos ecológicos de nicho de Taxus globosa.}

Geographic variables used to obtain the ecological niche models of Taxus globosa.

Variables climáticas (Worldclim 1.4, Hijmans et al. 2005)

1. Temperatura promedio anual $\left({ }^{\circ} \mathrm{C}\right)$

2. Oscilación diurna de la temperatura $\left({ }^{\circ} \mathrm{C}\right)$

3. Isotermalidad $\left({ }^{\circ} \mathrm{C}\right)$

4. Estacionalidad de la temperatura $\left({ }^{\circ} \mathrm{C}\right)$

5. Temperatura máxima promedio del periodo más cálido $\left({ }^{\circ} \mathrm{C}\right)$

6. Temperatura mínima promedio del periodo más frío $\left({ }^{\circ} \mathrm{C}\right)$

7. Oscilación anual de la temperatura $\left({ }^{\circ} \mathrm{C}\right)$

8. Temperatura promedio del trimestre más lluvioso $\left({ }^{\circ} \mathrm{C}\right)$

9. Temperatura promedio del trimestre más seco $\left({ }^{\circ} \mathrm{C}\right)$

10. Temperatura promedio del trimestre más cálido $\left({ }^{\circ} \mathrm{C}\right)$

11. Temperatura promedio del trimestre más frío $\left({ }^{\circ} \mathrm{C}\right)$

12. Precipitación anual $(\mathrm{mm})$

13. Precipitación del periodo más lluvioso $(\mathrm{mm})$

14. Precipitación del periodo más seco $(\mathrm{mm})$

15. Estacionalidad de la precipitación $(\mathrm{mm})$

16. Precipitación del trimestre más lluvioso $(\mathrm{mm})$

17. Precipitación del trimestre más seco $(\mathrm{mm})$

18. Precipitación del trimestre más cálido $(\mathrm{mm})$

19. Precipitación del trimestre más frío $(\mathrm{mm})$

Variables topográficas (Hydro $1 \mathrm{k}$ )

1. Modelo digital de elevación

2. Pendiente

3. Índice topográfico 
Para evitar el sesgo en la elaboración de los modelos por la selección de los puntos de entrenamiento (datos para generar los modelos) y los puntos de evaluación, se realizaron 100 réplicas utilizando la opción para aleatorizar la selección de los datos. Se utilizó el $70 \%$ de los registros puntuales de la especie para generar los modelos y el restante $30 \%$ como datos para la evaluación, y en cada réplica se utilizó validación cruzada que consiste en replicar los datos para la elaboración de los modelos y esos mismos datos sirvieron para evaluar el siguiente modelo. Los parámetros utilizados para obtener dichos modelos fueron multiplicador de regularización (regularization multiplier) $=1$, número máximo de puntos de fondo (maximum number of background points) $=10000$, límite de convergencia (convergence limit $)=0.00001 \mathrm{y}$ número máximo de repeticiones (maximum iterations) $=500$. Los mapas resultantes se exportaron al programa ArcView 3.2 (ESRI 1999). Se utilizó el estadístico I (Warren et al. 2008) para cuantificar el grado de similitud entre los 100 modelos realizados por medio del programa ENMTools 1.1.

Los valores del estadístico I asumen que no existen diferencias significativas entre todas las réplicas, por lo que la selección del modelo final se hizo a partir de los 10 modelos con valores más altos del área bajo la curva (AUC) obtenidos directamente de la evaluación de los modelos por medio de las curvas ROC. A partir de estos se estableció un valor umbral de presencia que maximiza los valores de probabilidad de los datos puntuales de presencia y los que se generan a partir de las pseudoausencias (Jiménez-Valverde \& Lobo 2007; en el software la opción a la que corresponde es "maximum sensitivity plus specificity"), seleccionando finalmente el modelo que presentó los errores de omisión más bajos.

Se realizó un análisis entre las variables utilizadas donde se encontró que ocho de estas presentan una alta correlación en el área de estudio (variables $1,3,7$, $10,11,12,16$ y 17, ver Tabla 1 ). Como prueba, se realizaron modelos eliminando estas variables y fueron comparados contra los que incluyeron todas. Posteriormente se verificó si existían diferencias significativas entre ambos conjuntos de modelos utilizando el índice I mediante el programa ENMTools 1.1 (Warren et al. 2008), demostrando que las diferencias entre ambos conjuntos de modelos no son significativas (I 0.8 ).

Una vez que se genera el modelo de nicho ecológico, es necesario proyectarlo a lo que se define como el área de distribución geográfica potencial, debido a que el modelo de nicho puede estar sobrestimando el área de distribución geográfica de la especie (Illoldi \& Escalante 2008), ya que en el modelo se están considerando todos aquellos sitios en donde se presentan las mismas condiciones ecológicas que en los sitios de recolecta originales, pero se puede incurrir en el error de incluir áreas donde históricamente se sabe que la especie no existe (Navarro-Siguienza et al. 2003). Por lo anterior, se han generado métodos para proyectar el modelo de nicho ecológico al área de distribución geográfica potencial, eliminando de la sobrepredicción aquellos sitios donde se conoce que el taxón no puede estar o no ha sido registrado mediante un recorte a partir de esquemas de regionalización desarrollado previamente (Illoldi \& Escalante 2008) como lo han llevado a cabo NavarroSigüenza et al. (2003), Soberón \& Peterson (2005) y Ríos-Muñoz \& Navarro-Sigüenza (2009), entre otros. En este estudio, para pasar del modelo de nicho ecológico hacia una representación de la distribución geográfica potencial de Taxus globosa, se utilizó la regionalización biogeográfica de México de Arriaga et al. (1997). Los datos de las localidades de recolecta se utilizaron para seleccionar las provincias biogeográficas donde se distribuye la especie (Peterson \& Vieglais 2001, Navarro-Sigüenza et al. 2003, Illoldi-Rangel \& Escalante 2008).

A partir de la distribución potencial de la especie, se utilizaron tres escenarios de cambio de uso de suelo para tres épocas, 1976, 1996 y 2000 para eliminar aquellas áreas que han sido modificadas antropogénicamente y donde no puede encontrarse la especie al estar asociada a zonas de hábitat conservado. Las áreas que fueron eliminadas de las áreas de distribución potencial por ser inapropiadas corresponden a zonas urbanas, agrícolas, pecuarias, forestales, sin vegetación aparente y pastizales inducidos (Ríos-Muñoz \& Navarro-Sigüenza 2009), observando las diferencias al comparar la superficie de distribución potencial para la especie con datos de las últimas tres décadas. Los escenarios de cambio de uso de suelo se generaron con información de 1973 a 1976 basada en fotografía aérea y los inventarios nacionales forestales de 1996 y 2000 (Velázquez et al. 2002).

Por último, considerando el escenario más reciente (2000) y con ayuda del programa ArcView 3.2 (ESRI 1999) se sobrepuso el modelo generado con Maxent de la distribución potencial remanente de la especie con los mapas digitales de las Áreas Naturales Protegidas Federales (ANPs) (CONANP 2007) y con las Regiones Terrestres Prioritarias para la conservación (RTPs) propuestas por la Comisión Nacional para el uso y el conocimiento de la Biodiversidad (Arriaga et al. 2000), para calcular el porcentaje de distribución y con el fin de evaluar su representatividad en ambos programas de conservación. En ambos casos se calculó el área de distribución potencial tomando el remanente del año 2000 para poder determinar el porcentaje de la distribución potencial bajo protección o en planes para protegerse. Las RTPs son áreas que albergan una gran riqueza de especies y ecosistemas en relación a otras áreas de México y presentan una integridad ecológica funcional donde existen oportunidades reales de conservación; dichas áreas fueron propuestas por un grupo de investigadores expertos en la diversidad biológica mexicana y fueron seleccionadas con base en su alto nivel de endemismo y riqueza de especies (Arriaga et al. 2000).

Debido a que se conoce poco del área de distribución geográfica y del tamaño de las poblaciones de muchas especies mexicanas en alguna categoría de riesgo (Luna et al. 2006), la amplitud de la distribución geográfica de las especies ha sido un factor decisivo para incluirlas en la Norma Oficial Mexicana vigente NOM-059 (SEMARNAT 2002), siendo este criterio espacial parte del método de evaluación de riesgo (MER) de extinción de las especies silvestres de México (SEMARNAT 2002).

\section{RESULTADOS}

Existe material herborizado depositado en las colecciones citadas de Taxus globosa para los estados de Chiapas, Hidalgo, Nuevo León, Oaxaca, Puebla, Querétaro, San Luis Potosí, Tamaulipas y Veracruz. Además, la especie fue 
mencionada para el Estado de México por Standley (1926), pero no existen ejemplares de herbario que respalden esta información; no obstante el modelo del nicho ecológico predice su presencia en el estado (Fig. 1). Esta especie se distribuye principalmente en la Sierra Madre Oriental, la porción este de la Faja Volcánica Transmexicana, la Sierra Norte de Oaxaca y Los Altos de Chiapas.

Las localidades donde Taxus globosa ha sido registrada fueron contrastadas $y$ comparadas con el modelo generado por Maxent mostrando un poder predictivo de un $94.5 \%$ y probado mediante la creación de curvas ROC (AUC = 0.997), mostrando que se trata de un muy buen modelo.

La distribución potencial de Taxus globosa para México sugiere la existencia de 35259 $\mathrm{km}^{2}$ sin considerar el impacto causado por el cambio de uso de suelo. Al considerar el escenario de 1976 quedaba un área de 26620 $\mathrm{km}^{2}$ (reducción del $24.5 \%$ de la vegetación original sin considerar la intervención humana), para 1996 se calculan $6847 \mathrm{~km}^{2}$ (reducción del $80.58 \%$ ) y para 2000 se estiman $6297 \mathrm{~km}^{2}$ (reducción del 82.14 \%) (Figs. 2 y 3). Lo anterior muestra una disminución drástica en el porcentaje de cobertura forestal de hasta el $80 \%$ para la especie (Fig. 2).

El cálculo de área para las ANPs únicamente cuenta con $744 \mathrm{~km}^{2}$, que representa el $11.8 \%$ del área potencial para 2000 distribuida en diez ANPs a lo largo de la Sierra Madre Oriental y Sierra Madre de Oaxaca (Fig. 4); el área potencial que se encuentra en Chiapas no cuenta con ningún área natural federal protegida que la incluya. Por otro lado, en el caso de las RTPs es de $3052 \mathrm{~km}^{2}$, que es equivalente al $48.46 \%$ del área potencial para 2000; esta área se encuentra repartida en 15 RTPs a lo largo del área de distribución (Fig. 5).

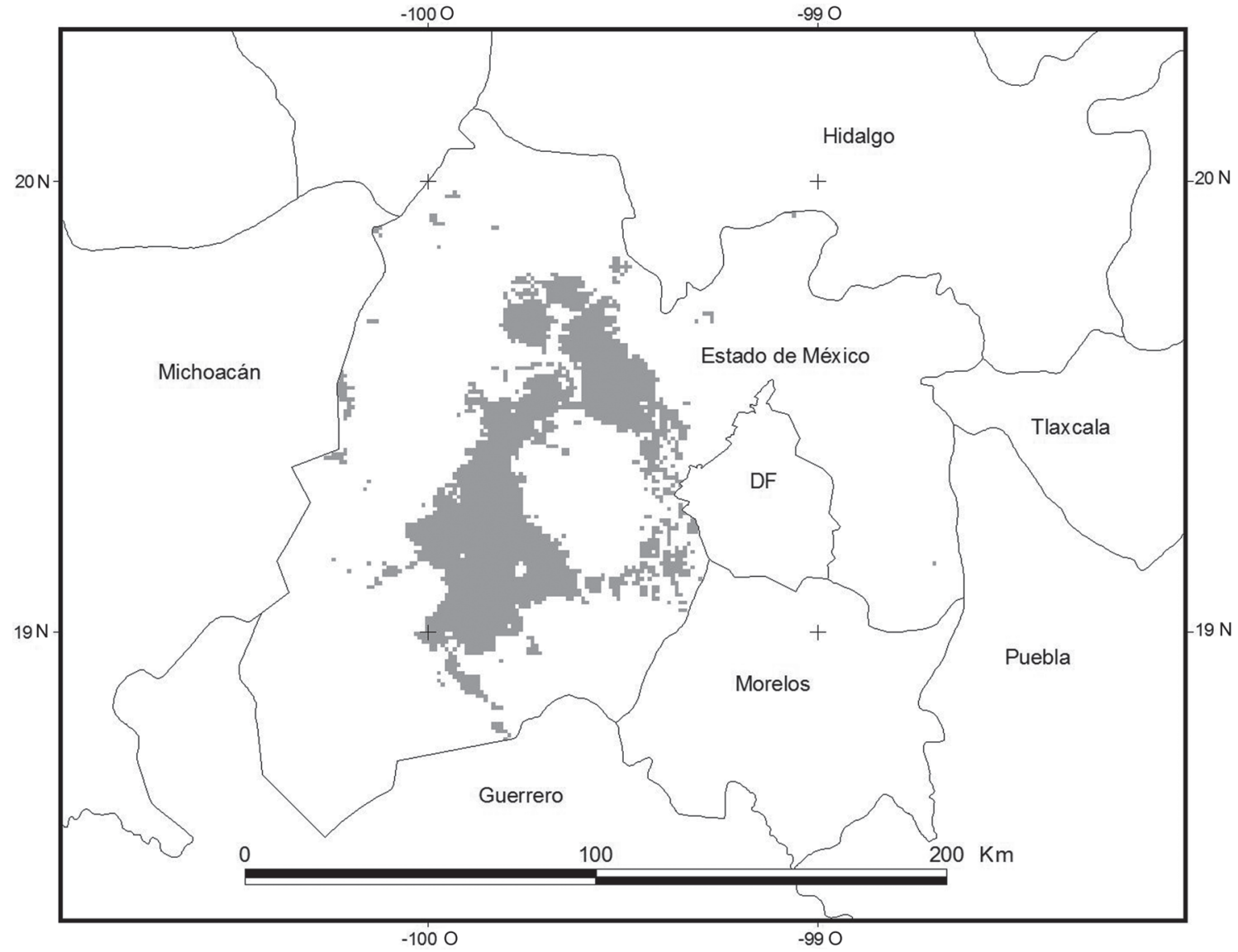

Fig. 1: Distribución geográfica potencial de Taxus globosa para el Estado de México. 


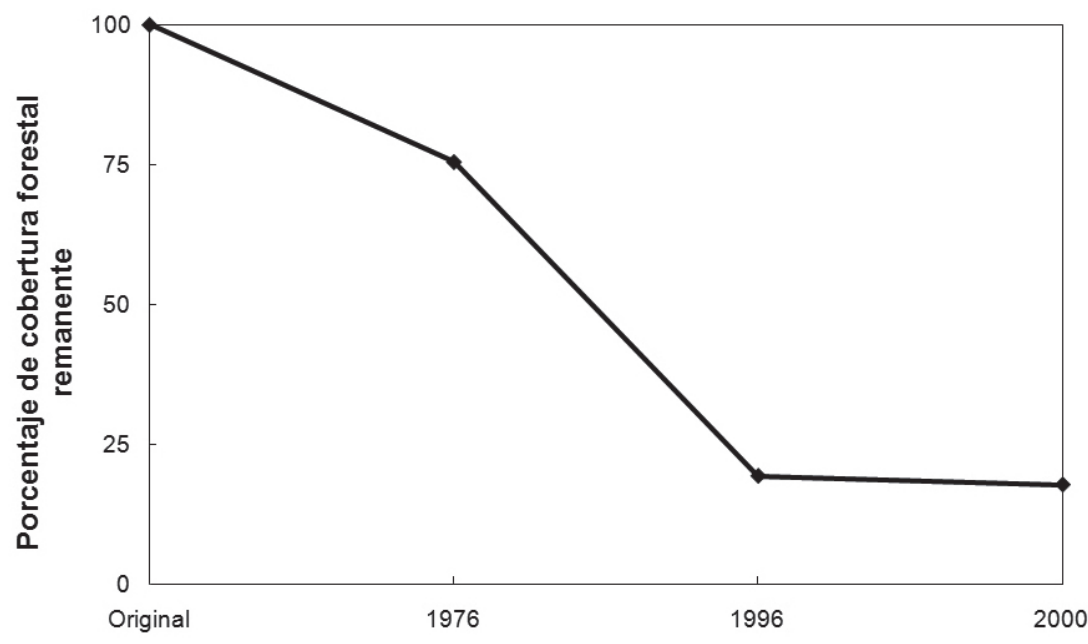

Años correspondientes a la modificación de cobertura vegetal

Fig. 2: Modificación del área de distribución geográfica potencial para Taxus globosa en México, bajo los escenarios de cambio de uso de suelo.

Modification of the geographic potential distribution for Taxus globosa in Mexico under different land use change scenarios.
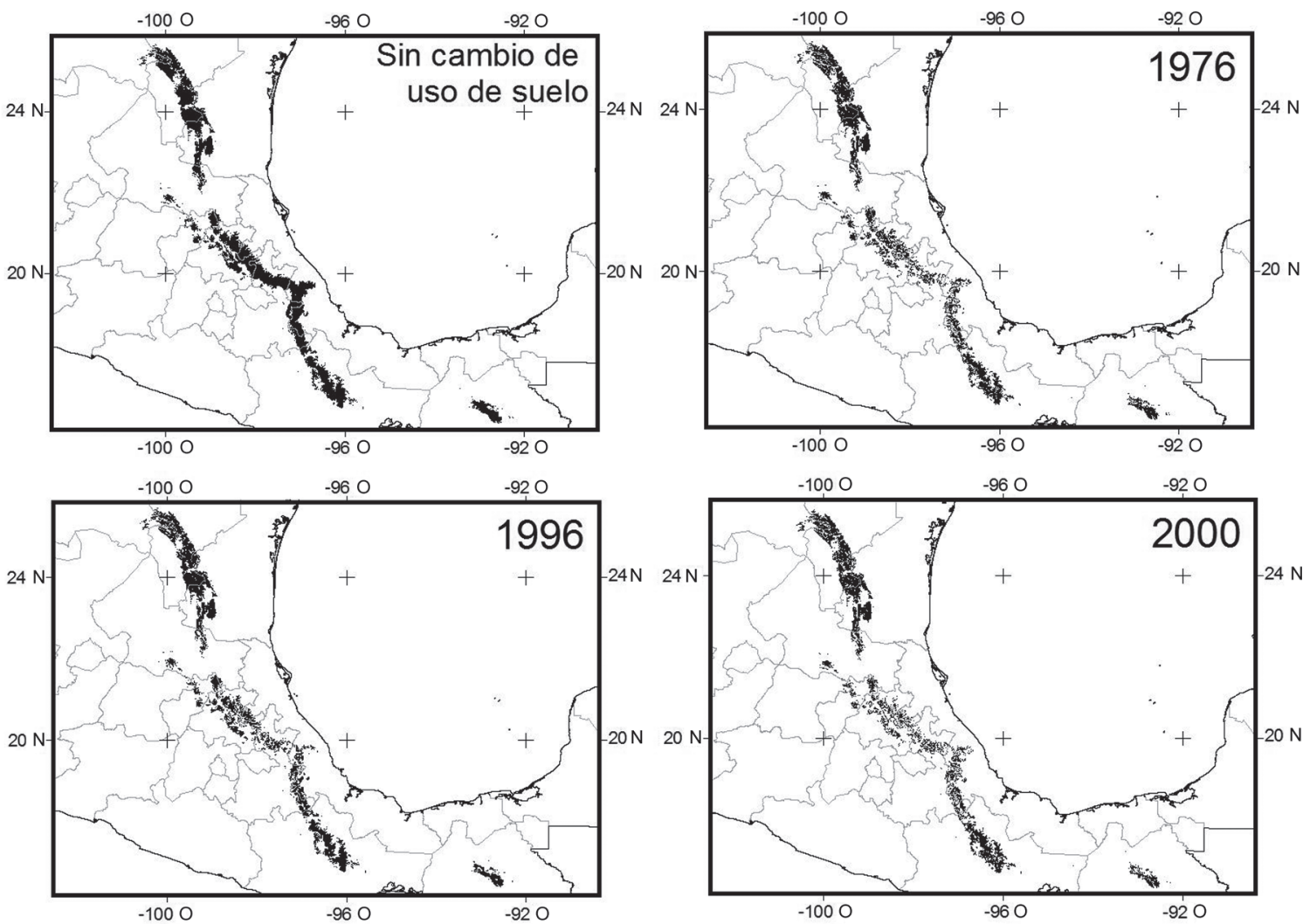

Fig. 3: Áreas de distribución geográfica potencial para Taxus globosa en México sin considerar el cambio de uso de suelo y considerando los tres escenarios (1976, 1996, 2000).

Areas of geographic potential distribution for Taxus globosa in Mexico excluding land use change and considering three different scenarios $(1976,1996,2000)$. 


\section{DISCUSIÓN}

Los resultados obtenidos sugieren que el modelo de distribución potencial de Taxus globosa coincide bastante bien con la distribución conocida de la especie, con algunas excepciones, lo cual refleja un porcentaje bajo de error de omisión (6\%). Aunque dentro del área de estudio existen variables ambientales altamente correlacionadas, la elaboración de los modelos mostró que estas no modifican de manera significativa la representación geográfica del nicho ecológico de la especie, por lo que los resultados presentados para Taxus globosa no se modifican al considerar los conjuntos de variables completas o eliminando las que se encuentran altamente correlacionadas. Esta especie habita en el noreste, centro y sur de México, así como en algunos bosques húmedos de Guatemala, Honduras y El Salvador. Si bien existe una baja incertidumbre, los mapas resultantes pueden usarse como base para proponer trabajo de campo en sitios específicos, como es el caso de algunas áreas prioritarias para la conservación y áreas naturales protegidas, donde la presencia de la especie ha sido sugerida por el modelo, pero que no se tiene evidencia directa de su existencia; asimismo, se pueden proponer sitios específicos con condiciones abióticas adecuadas para su crecimiento y desarrollo con propósitos de propagación y conservación.

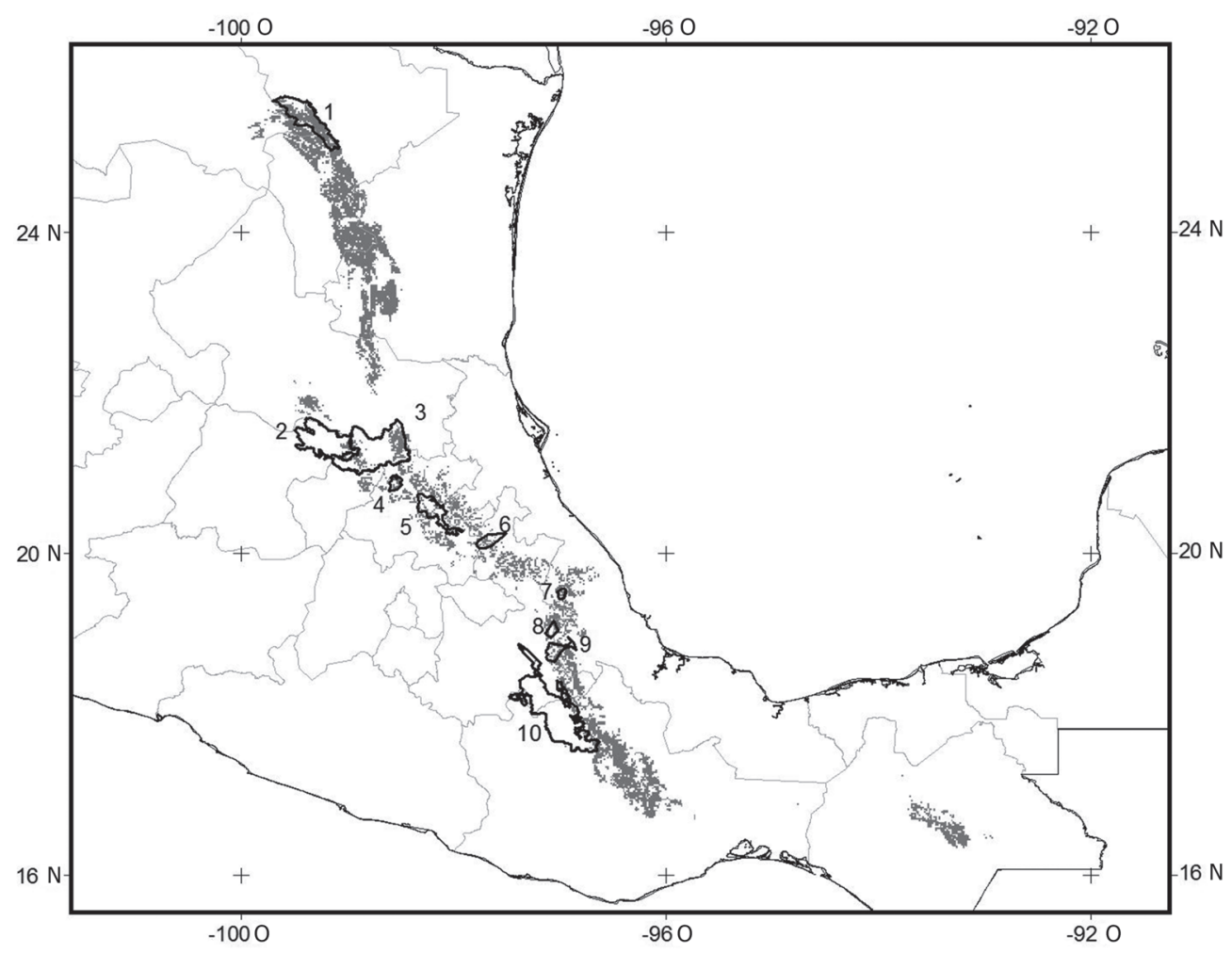

Fig. 4: Distribución geográfica potencial de Taxus globosa considerando el escenario de cambio de uso de suelo 2000 y su relación con las áreas naturales protegidas federales (ANPs). (1) Cumbres de Monterrey, (2) Sierra Gorda de Guanajuato, (3) Sierra Gorda, (4) Los Mármoles, (5) Barrancas de Metztitlán, (6) Cuenca Hidrográfica del Río Necaxa, (7) Cofre de Perote, (8) Pico de Orizaba, (9) Cañón de Río Blanco, (10) Valle de Tehuacán-Cuicatlán.

Geographic potential distribution of Taxus globosa under land use change scenario of year 2000 and its relationship with the Mexican natural protected areas (ANPs). (1) Cumbres de Monterrey, (2) Sierra Gorda de Guanajuato, (3) Sierra Gorda, (4) Los Mármoles, (5) Barrancas de Metztitlán, (6) Cuenca Hidrográfica del Río Necaxa, (7) Cofre de Perote, (8) Pico de Orizaba, (9) Cañón de Río Blanco, (10) Valle de Tehuacán-Cuicatlán. 
Solamente mediante trabajo de campo será posible detectar en cuánto el modelo generado sobreestima el área de distribución potencial más allá de sus límites reales. En este sentido, al sobreponer el mapa de distribución potencial de Taxus globosa con el mapa del sistema de áreas naturales protegidas federales (ANPs), se observó que buena parte del área (92\%) no está incluida dentro de los límites de estas áreas. El modelo muestra que solamente diez de estas áreas protegidas mexicanas incluyen poblaciones de la especie y solamente en estos casos se puede suponer que la especie está adecuadamente protegida, por lo que consideramos que la especie está mal representada en el sistema actual de áreas naturales protegidas federales de México. En el caso de las RTPs la representación es más alta, de casi un $50 \%$ del área potencial; el inconveniente es que estas últimas áreas no están reguladas por las leyes federales y no son oficiales.

Debido a que se predice su presencia en algunas áreas naturales protegidas, es imprescindible emprender salidas de campo para verificar su existencia y proponer planes de manejo y conservación de la especie en estas áreas. Desgraciadamente, como ocurre en otros países latinoamericanos, el que las poblaciones de una especie estén incluidas dentro de áreas protegidas no garantiza su conservación, como han sugerido previamente varios autores (Vanclay et al. 2001, Jackson et al. 2009).

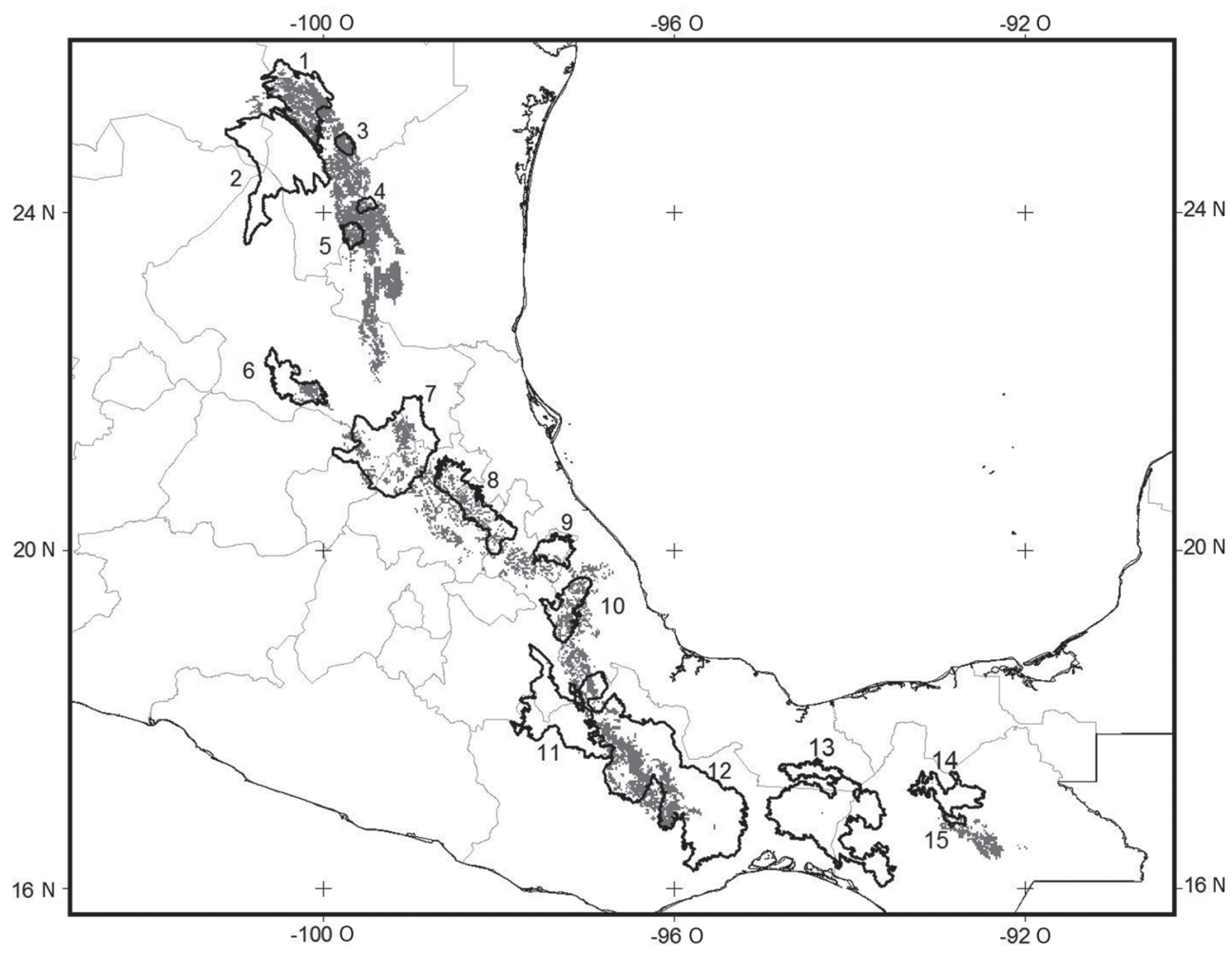

Fig. 5: Distribución geográfica potencial del Taxus globosa considerando el escenario de cambio de uso de suelo 2000 y su relación con las regiones terrestres prioritarias (RTPs). (1) El Potosí-Cumbres de Monterrey, (2) El Tokio, (3) Cañón de Iturbide, (4) Puerto Purificación, (5) San Antonio-Peña Nevada, (6) Sierra de Álvarez, (7) Sierra Gorda-Río Moctezuma, (8) Bosques Mesófilos de la Sierra Madre Oriental, (9) Cuetzalan, (10) Pico de Orizaba-Cofre de Perote, (11) Valle de Tehuacán-Cuicatlán, (12) Sierras del Norte de Oaxaca-Mixe, (13) Selva Zoque-La Sepultura, (14) Bosques Mesófilos de Los Altos de Chiapas, (15) Huitepec-Tzontehuitz.

Geographic potential distribution of Taxus globosa under land use change scenario of year 2000 and its relationship with the Mexican Terrestrial Priority Regions (RTPs). (1) El Potosí-Cumbres de Monterrey, (2) El Tokio, (3) Cañón de Iturbide, (4) Puerto Purificación, (5) San Antonio-Peña Nevada, (6) Sierra de Álvarez, (7) Sierra Gorda-Río Moctezuma, (8) Bosques Mesófilos de la Sierra Madre Oriental, (9) Cuetzalan, (10) Pico de Orizaba-Cofre de Perote, (11) Valle de TehuacánCuicatlán, (12) Sierras del Norte de Oaxaca-Mixe, (13) Selva Zoque-La Sepultura, (14) Bosques Mesófilos de Los Altos de Chiapas, (15) Huitepec-Tzontehuitz. 
La eficacia del modelo generado con Maxent en la predicción de la distribución geográfica de Taxus globosa demuestra la habilidad del mismo para estimar la distribución de esta especie en condiciones reales. Esta credibilidad aumentará cuando en los sitios donde se predice su presencia se encuentre la especie, como es el caso del Estado de México, donde ya fue mencionada su presencia anteriormente por Standley (1926), pero que no existen ejemplares de respaldo en ningún herbario de México o el extranjero. En el caso del estado de Puebla solamente existen ejemplares de herbario recolectados en 1908 por C. H. Pringle \#10808 (IBUG, XAL) en la localidad de Honey, y desde aquel tiempo la especie no ha sido colectada de nuevo.

Es posible que los factores climáticos, influenciados por la variación altitudinal, sean decisivos para la existencia de Taxus globosa en algunas áreas señaladas por el modelo, ya que es una especie asociada a las áreas montañosas de Mesoamérica (ContrerasMedina \& Luna 2001) y es considerada bajo la categoría de montana al habitar preferentemente en un intervalo altitudinal entre los 1000-2600 msnm (Contreras-Medina 2004). Al parecer la distribución de esta especie en parte está influenciada por factores históricos que se demuestran por su presencia en la parte noreste y sur de México, además de la parte norte de América Central y que según Rzedowski (1978), es una de las especies de distribución discontinua que establecen una relación florística con el este de América del Norte (Estados Unidos y Canadá) y que forman parte del bosque caducifolio en los Estados Unidos y del bosque nublado de México y América Central (Zavala-Chávez et al. 2001), como ocurre con otras especies de plantas (e.g., Fagus grandifolia Ehrh., Liquidambar styraciflua L. y Pinus strobus L., entre otras). También la combinación de factores históricos junto con otros de índole ambiental y ecológico determinan su ocupación, como ha sido sugerido para otros taxa y asociaciones vegetales (Pearson \& Dawson 2003). A partir de observaciones directas en campo en los estados de Hidalgo y Oaxaca, además de la información extraída de las etiquetas de herbario, la especie puede ser considerada como escasa, salvo las poblaciones que se encuentran en la Sierra Gorda del estado de Querétaro, en las cuales se refiere a la especie como localmente abundante (Zamudio 1992). De acuerdo con Zavala-Chávez et al. (2001), la regeneración parece basarse en la producción de semillas, pero en algunos lugares se ha encontrado una notable producción de rebrotes a partir de la base del tallo, característica que podría ser aprovechada para su propagación $y$ regeneración artificial en México (ZavalaChávez et al. 2001), actividad a desarrollar fundamentalmente en aquellas áreas naturales protegidas donde se conoce la presencia de la especie, como es el caso de Calocedrus decurrens (Torrey) Florin, otra gimnosperma amenazada y que se cultiva dentro del Parque Nacional Constitución de 1857, donde también crece de manera natural en el estado de Baja California, México.

La habilidad para predecir eficazmente la distribución de cualquier especie depende básicamente de la cantidad y la calidad de los datos con los que se cuente (Peterson et al. 2002). Varios autores (Lindenmayer et al. 1996, Peterson et al. 2000, Stockwell \& Peterson 2002) han sugerido el uso de un mínimo de 50 localidades debidamente georreferenciadas para que los análisis predictivos sean robustos y en nuestro caso se utilizó un número mayor, por lo que se cumple con este requisito. La resolución espacial de los parámetros ambientales que incluyen las coberturas geográficas es otro factor que debe tomarse en cuenta en estos análisis (Peterson et al. 2002). En el presente trabajo se utilizó una resolución espacial fina (pixeles de $1 \mathrm{~km}^{2}$ ), la cual permite tener una buena aproximación de las condiciones del paisaje presentes en el país y hacer algunas inferencias sobre lo que le ha sucedido al hábitat (Peterson et al. 2006). Sin embargo, los modelos de cambio de uso de suelo utilizados en este estudio han sido generados en distintos momentos y a partir de métodos diferentes, por lo que la compatibilidad a pesar de tratar de ser lo más completa posible, no pudo ser total (Velázquez et al. 2002, Peterson et al. 2006). Estas diferencias hacen que los modelos presenten algunas limitantes a nivel espacial, pues solo son adecuados al considerar lo que sucede a escala nacional y en patrones generales. A escalas más locales pueden representar 
problemas, ya que las delimitaciones de tipos de uso de suelo a escala muy fina no coinciden en su totalidad, por lo que pueden representar información que no se ajuste totalmente con la realidad (Ríos-Muñoz \& Navarro-Sigüenza 2009).

Muchas otras especies consideradas como características o diagnósticas de los bosques nublados de México (o bosque mesófilo de montaña sensu Rzedowski 1978) habitan en condiciones climáticas y ambientales similares, por lo cual los modelos generados son útiles para detectar con certeza los requerimientos ecológicos indispensables para asegurar la existencia y promover la conservación de ciertos parches de este tipo de vegetación en México, el cual por sí mismo se considera como amenazado (Churchill et al. 1995, Luna et al. 2000).

Debido a la alta concentración de especies con distribución muy restringida en los bosques mesófilos de montaña de México (como Bernardia mollis Lundell, Bouvardia xylostoides Hook. et Arn., Ceratozamia kuesteriana Regel, Deppea hernandezii Lorence y Nopalxochia phyllanthoides (DC) Britton et Rose, entre otras) y a que este tipo de vegetación ha sido fuertemente afectado por las actividades humanas en las últimas décadas, se considera que la conservación de los parches de este bosque garantiza en cierta forma el mantenimiento de las poblaciones de algunas de estas especies, evitando la desaparición de especies endémicas. La selección de algunas especies asociadas a este tipo de vegetación (ya sean diagnósticas o endémicas) es importante para el mantenimiento del hábitat y también para proponer un plan integral de áreas protegidas en países megadiversos, como es el caso de México. El utilizar un algoritmo como Maxent aplicado a los datos de distribución de Taxus globosa, especie que está considerada como rara y amenazada, debido a que actualmente existen escasas poblaciones compuestas por pocos individuos, nos permitió generar con mayor precisión y robustez la distribución potencial de la especie.

Actualmente la información disponible para establecer estrategias y acciones sobre la conservación de las plantas mexicanas bajo alguna categoría de riesgo ha consistido principalmente en estudios demográficos $\mathrm{y}$ ecológicos de estas especies (e.g., Sosa et al. 1998, Martínez-Ávalos et al. 2004), colecta de semillas y propagación vegetativa para conservación ex situ en jardines botánicos (e.g., Sánchez et al. 1995, Vovides 1995) y reconocimiento de áreas donde se concentran estas especies con la finalidad de incluirlas en futuros planes de conservación (Luna et al. 2006, Contreras-Medina \& Luna 2007), así como estudios puntuales sobre la biología de algunos taxa (Martínez-Ávalos et al. 2004, Téllez-Valdés et al. 2006, Solano \& Feria 2007, Yberri 2009). Sin embargo, otros factores que afectan también a las poblaciones naturales de plantas han sido poco evaluados, como el cambio climático (Téllez-Valdés \& DávilaAranda 2003, Gómez-Mendoza \& Arriaga 2007) o ha sido nulo, como el grado de modificación del hábitat, por lo que el presente estudio representa una propuesta y una contribución a la preservación de las plantas mexicanas amenazadas.

El panorama poco alentador que se observa a partir de los datos generados por el cambio de uso de suelo en las áreas donde habita Taxus globosa en México, requiere de la necesidad de hacer una evaluación de las poblaciones y los requerimientos particulares de la especie, así como el estudio del hábitat para su cultivo y propagación, con la finalidad de apoyar la planeación y evaluación de acciones de conservación complementarias que conlleven a mantener poblaciones viables de la especie a largo plazo.

\section{AGRADECIMIENTOS}

Agradecemos a Enrique Martínez Meyer, Adolfo Navarro y dos revisores anónimos por la lectura crítica y los comentarios al manuscrito. Carlos Ruiz y Alberto González colaboraron en la obtención de las coordenadas de los mapas. También se agradece a los curadores y personal de los herbarios citados en el texto, quienes amablemente permitieron la revisión de los ejemplares en sus colecciones. Asimismo CONACyT y DGAPA-PAPIIT apoyaron este trabajo a través de los proyectos SEMARNAT-2004-C01-311 e IN209108 respectivamente.

\section{LITERATURA CITADA}

ARRIAGA L, C AGUILAR, D ESPINOSA \& R JIMÉNEZ (eds) (1997) Regionalización ecológica y biogeográfica de México. Taller desarrollado en 
la Comisión Nacional para el Conocimiento y Uso de la Biodiversidad (CONABIO). CONABIO. Ciudad de México, México.

ARRIAGA L, JM ESPINOZA, C AGUILAR, E MARTINEZ, L GOMEZ \& E LOA (coord) (2000) Regiones terrestres prioritarias de México. Comisión Nacional para el Conocimiento y Uso de la Biodiversidad (CONABIO). CONABIO, Ciudad de México, México.

BROOKS TM, RA MITTERMEIER, CG MITTERMEIER, GAB DA FONSECA, AB RYLANDS et al. (2001) Habitat loss and extinction in the hotspots of biodiversity. Conservation Biology 16: 909-923.

CONANP (2007) Comisión Nacional de Áreas Naturales Protegidas. URL: http://www.conanp.gob.mx (accedido Diciembre 2, 2009).

CHURCHILL SP, H BALSLEV, E FORERO \& JL LUTEYN (eds) (1995) Biodiversity and conservation of neotropical montane forests. Proceedings of the Neotropical Montane Forest Biodiversity and Conservation Symposium. The New York Botanical Garden, Nueva York.

COLLAR NJ (1996) The reasons for red data books. Oryx 30: 121-130.

CONTRERAS-MEDINA R (2004) Gimnospermas. En: Luna I, JJ Morrone \& D Espinosa (eds) Biodiversidad de la Sierra Madre Oriental: Un enfoque multidisciplinario: 137-148. CONABIOUNAM, Ciudad de México.

CONTRERAS-MEDINA R \& I LUNA (2001) Presencia de Taxus globosa Schltdl. (Taxaceae) en el estado de Chiapas, México. Polibotánica 12: 5156.

CONTRERAS-MEDINA R \& I LUNA (2007) Species richness, endemism and conservation of Mexican gymnosperms. Biodiversity and Conservation 16: 1803-1821.

DÁVILA-ARANDA P, R LIRA \& J VALDÉS-REYNA (2004) Endemic species of grasses in Mexico: A phytogeographic approach. Biodiversity and Conservation 13: 1101-1121.

ELITH JCH, RP ANDERSON, M DUDIK, S FERRIER, A GUISAN et al. (2006) Novel methods improve prediction of species' distributions from occurrence data. Ecography 29: 129-151.

ENGLER R, A GUISAN \& L RECHSTEINER (2004) An improved approach for predicting the distribution of rare and endangered species from occurrence and pseudo-absence data. Journal of Applied Ecology 41: 263-274.

ESRI (1999) ArcView 3.2. ESRI (Environmental Scientific Research Institute). Redlands, California, USA.

GARCÍA-MENDOZA A (1995) Riqueza y endemismos de la familia Agavaceae en México. En: Linares E, P Dávila, F Chiang, R Bye \& T Elías (eds) Conservación de plantas en peligro de extinción: Diferentes enfoques: 51-75. Instituto de Biología, UNAM, Ciudad de México.

GARCÍA F \& P CASTILLO (2000) Aspectos ecológicos de Taxus globosa Schlecht. en las Mesas de San Isidro, municipio de Río Verde, San Luis Potosí. Biotam 11: 11-18.

GOLUBOV J, MC MANDUJANO \& F MANDUJANO (2005) Diversidad alfa y beta en Opuntia y Agave. En: Halffter G, J Soberón, P Koleff \& A Melic (eds) Sobre diversidad biológica: El significado de las diversidades alfa, beta y gamma: 221-230. CONABIO, CONACYT \&
DIVERSITAS, Zaragoza, España.

GÓMEZ-MENDOZA L \& L ARRIAGA (2007) Modeling the effect of climate change on the distribution of oak and pine species of Mexico. Conservation Biology 21: 1545-1555.

GONZÁLEZ-ÄVILA P, A TORRES-MIRANDA, I LUNA \& M VILLEGAS-RÍOS (en prensa) Patterns of diversity and endemism: An example with Mexican species of Echinoramaria. North American Fungi.

GROOMBRIDGE B (ed) (1992) Global biodiversity. Status of the Earth's living resources. Chapman \& Hall, Londres.

HEYWOOD VH \& SD DAVIS (1997) Introduction. En: Davis SD, VH Heywood, O Herrera-MacBryde, J Villa-Lobos \& AC Hamilton (eds) Centres of plant diversity. A guide and strategy for their conservation: 1-38. Vol. 3. The Americas. WWF/ IUCN, Cambridge.

HUTCHINSON GE (1957) Concluding remarks. Cold Spring Harbor Symposium on Quantitative Biology 22: 415-457.

ILLOLDI-RANGEL P \& T ESCALANTE (2008) De los modelos de nicho ecológico a las áreas de distribución geográfica. Biogeografía 3: 7-12.

JACKSON SF, K WALKER \& KJ GASTON (2009) Relationship between distributions of threatened plants and protected areas in Britain. Biological Conservation 142: 1515-1522.

JIMÉNEZ-VALVERDE A \& JM LOBO (2007) Threshold criteria for conversion of probability of species presence to either-or presenceabsence. Acta Oecologica 31: 361-369.

LINDENMAYER DB, BG MACKEY \& HA NIX (1996) The bioclimatic domains of four species of commercially important eucalypts from southeastern Australia. Australian Forestry 59: 74-89.

LUNA I \& R CONTRERAS-MEDINA (2010) Plant biodiversity hotspots and biogeographic methods. En: Rescigno V \& S Maletta (eds) Biodiversity hotspots 181-191. Nova Science Publishers, Nueva York.

LUNA I, O ALCÁNTARA, D ESPINOSA \& JJ MORRONE (2000) Track analysis and conservation priorities in the cloud forests of Hidalgo, Mexico. Diversity and Distributions 6: 137-143.

LUNA I, O ALCÁNTARA, R CONTRERAS-MEDINA \& A PONCE (2006) Biogeography, current knowledge and conservation of threatened vascular plants characteristic of Mexican temperate forests. Biodiversity and Conservation 15: 3773-3799.

LUNA I, O ALCÁNTARA \& R CONTRERAS-MEDINA (en prensa) Threatened temperate plant species: contributions to their biogeography and conservation in Mexico. En: Tepper GH (ed) Species diversity and extinction. Nova-Science Publishers. New York.

MARTÍNEZ-ÁVALOS JG, MC MANDUJANO, J GOLUBOV, M SOTO \& V JOHANNES (2004) Análisis del método de evaluación de riesgo (MER) del "falso peyote" (Astrophytum asterias (Zucc) Lem.) en México. Cactáceas y Suculentas Mexicanas 49: 118-127.

MITTERMEIER RA \& CG MITTERMEIER (1992) La importancia de la diversidad biológica de México. En: Sarukhán J \& R Dirzo (eds) México ante los retos de la biodiversidad: 63-73. CONABIO, Ciudad de México.

MYERS N, RA MITTERMEIER, CG MITTERMEIER, 
GAB DA FONSECA \& J KENT (2000) Biodiversity hotspots for conservation priorities. Nature 403: 853-858.

NAVARRO-SIGÜENZA A, AT PETERSON, YJ NAKAZAWA \& I LIEBIG-FOSSAS (2003) Colecciones biológicas, modelaje de nichos ecológicos y los estudios de la biodiversidad. En: Morrone JJ \& J Llorente (eds) Una perspectiva latinoamericana de la biogeografía: 115-122. UNAM-CONABIO, Ciudad de México.

NIX HA (1986) BIOCLIM, a bioclimatic analysis and prediction system. CSIRO Division Water and Land Resources Research Annual Report. CSIRO Publishing, Canberra, Australia.

PEARSON RG \& TP DAWSON (2003) Predicting the impacts of climate change on the distribution of species: Are bioclimatic envelope models useful? Global Ecology and Biogeography 12: 361-371.

PETERSON AT \& KP COHOON (1999) Sensivity of distributional prediction algorithms to geographic data completeness. Ecological Modelling 117: 159-164.

PETERSON AT \& DA VIEGLAIS (2001) Predicting species invasions using ecological niche modeling: New approaches from bioinformatics attack a pressing problem. BioScience 51: 363371.

PETERSON AT, SL EGBERT, V SÁNCHEZ-CORDERO $\&$ KP PRICE (2000) Geographic analysis of conservation priority: Endemic birds and mammals in Veracruz, Mexico. Biological Conservation 93: 85-94.

PETERSON AT, LG BALL \& KP COHOON (2002) Predicting distributions of Mexican birds using ecological niche modelling methods. Ibis 144: 27-32.

PETERSON AT, V SÁNCHEZ-CORDERO, E MARTÍNEZ-MEYER \& A NAVARRO-SIGÜENZA (2006) Tracking population extirpations via melding ecological niche modeling with landcover information. Ecological Modeling 195: 229236.

PHILLIPS SJ, M DUDIK \& RE SCHAPIRE (2004) A maximum entropy approach to species distribution modeling. En: Proceedings of the $21^{\text {st }}$ International Conference on Machine Learning, Banff, Canadá.

PHILLIPS SJ, RP ANDERSON \& RE SCHAPIRE (2006) Maximum entropy modeling of species geographic distributions. Ecological Modelling 190: 231-259.

RAMÍREZ-BARAHONA S, A TORRES-MIRANDA, M PALACIOS-RÍOS \& I LUNA (2009) Historical biogeography of the Yucatan Peninsula, Mexico: A perspective from ferns (Monilophyta) and lycopods (Lycophyta). Biological Journal of the Linnean Society 98: 775-786.

RAPOPORT EH (1975) Areografía: Estrategias geográficas de las especies. Fondo de Cultura Económica, Ciudad de México.

RÍOS-MUÑOZ CA \& A NAVARRO-SIGÜENZA (2009) Efectos del cambio de uso de suelo en la disponibilidad hipotética de hábitat para los psitácidos de México. Ornitología Neotropical 20: 491-509.

RZEDOWSKI J (1978) Vegetación de México. Limusa, Ciudad de México.

SÁNCHEZ E, G GALINDO \& J HERNÁNDEZ (1995) Propagación de cactáceas del estado de Querétaro, México: Estrategia para su conservación. En: Linares E, P Dávila, F Chiang, R Bye \& T Elías (eds) Conservación de plantas en peligro de extinción: Diferentes enfoques: 107-115. Instituto de Biología, UNAM, Ciudad de México, México.

SÁNCHEZ-CORDERO V, P ILLOLDI-RANGEL, MA LINAJE, S SARKAR \& AT PETERSON (2005) Deforestation and extant distributions of Mexican endemic mammals. Biological Conservation 126: 465-473.

SEMARNAT (2002) Norma Oficial Mexicana NOM-059ECOL-2001, Protección ambiental -especies nativas de México y de flora y fauna silvestrescategorías de riesgo y especificaciones para su inclusión, exclusión o cambio- lista de especies en riesgo. Secretaría de Medio Ambiente y Recursos Naturales. Diario Oficial de la Federación (México): 1-80.

SKOV F (2000) Potential plant distribution mapping based on climatic similarity. Taxon 49: 503-515.

SOBERON J \& AT PETERSON (2005) Interpretation of models of fundamental ecological niches and species' distributional areas. Biodiversity Informatics 2: 1-10.

SOLANO E \& P FERIA (2007) Ecological niche modeling and geographic distribution of the genus Polianthes L. (Agavaceae) in Mexico: Using niche modeling to improve assessments of risk status. Biodiversity and Conservation 16: 1885-1900.

SOSA V, AP VOVIDES \& G CASTILLO-CAMPOS (1998) Monitoring endemic plant extinction in Veracruz, Mexico. Biodiversity and Conservation 7: 1521-1527.

STANDLEY PC (1926) Taxaceae. En: Trees and shrubs of Mexico. Contributions from the United States National Herbarium 23: 50-51.

STOCKWELL DRB \& D PETERS (1999) The GARP modeling system: Problems and solutions to automated spatial prediction. International Journal of Geographic Information Science 13: 143-158.

STOCKWELL DRB \& AT PETERSON (2002) Effects of sample size on accuracy of species distribution models. Ecological Modelling 148: 1-13.

TÉLLEZ-VALDÉS O \& P DÁVILA-ARANDA (2003) Protected areas and climate change: A case study of the cacti in the Tehuacán-Cuicatlán Biosphere Reserve, Mexico. Conservation Biology 17: 846-853.

TÉLLEZ-VALDÉS O, P DÁVILA-ARANDA \& R LIRASAADE (2006) The effects of climate change on the long-term conservation of Fagus grandifolia var. mexicana, an important species of the cloud forest in eastern Mexico. Biodiversity and Conservation 15: 1095-1107.

VANCLAY JK, AG BRUNER, RE GULLISON, RE RICE \& GAB DA FONSECA (2001) The effectiveness of parks. Science 293: 1007-1008.

VELÁZQUEZ A, JE MAS, JR DÍAZ-GALLEGOS, R MAYORGA-SAUCEDO, PC ALCÁNTARA et al. (2002) Patrones y tasas de cambio de uso de suelo en México. Gaceta Ecológica 62: 21-37.

VILLARREAL JA, J VALDÉS \& JL VILLASEÑOR (1996) Corología de las asteráceas de Coauhila, México. Acta Botanica Mexicana 36: 29-42.

VILLASEÑOR JL \& O TÉLLEZ-VALDÉS (2004) Distribución potencial de las especies del género Jefea (Asteraceae) en México. Anales del Instituto de Biología UNAM (Serie Botánica) 75: 205-220. 
VOVIDES AP (1995) Experiencias y avances en el conocimiento de las plantas mexicanas en peligro de extinción. En: Linares E, P Dávila, F Chiang, R Bye \& T Elías (eds) Conservación de plantas en peligro de extinción: Diferentes enfoques: 139-144. Instituto de Biología, UNAM, Ciudad de México, México.

WARREN DL, RE GLOR \& M TURELLI (2008) Environmental niche equivalency versus niche conservatism: Quantitative approaches to niche evolution. Evolution 62: 2868-2883.

YBERRI FG (2009) Distribución geográfica de Nopalxochia phyllanthoides (DC) Britton et Rose
(Cactaceae): Modelos predictivos y conservación. Tesis Profesional, Facultad de Ciencias, Universidad Nacional Autónoma de México, México DF.

ZAMUDIO S (1992) Familia Taxaceae. En: Rzedowski J \& G Calderón (eds) Flora del Bajío y de regiones adyacentes. Fascículo 9. Instituto de Ecología, Pátzcuaro, Michoacán, México.

ZAVALA-CHÁVEZ F, M SOTO-HERNÁNDEZ \& MT RODRÍGUEZ-GONZÁLEZ (2001) El romerillo (Taxus globosa Schlecht.): Biología, dificultades y perspectivas de su uso. Revista Chapingo Horticultura 7: 77-94. 
